\title{
Preoperative antithrombin supplementation in cardiac surgery: A randomized controlled trial
}

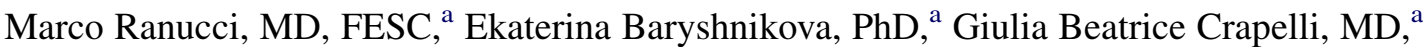 \\ Michael K. Woodward, ${ }^{\mathrm{b}}$ Antonio Paez, MD, ${ }^{\mathrm{b}}$ and Gabriele Pelissero, MD, PhD ${ }^{\mathrm{c}}$
}

\begin{abstract}
Objectives: Purified antithrombin supplementation in cardiac surgery has been suggested for the treatment of heparin resistance and the prevention of thromboembolic complications. This study is a randomized controlled trial of preoperative purified antithrombin supplementation, with the primary end point of avoiding low $(<58 \%)$ postoperative antithrombin activity levels and secondary end points including avoidance of heparin resistance, clinical outcome, and safety end points.
\end{abstract}

\begin{abstract}
Methods: Two hundred patients were randomly allocated to the antithrombin group and the control group. Patients in the antithrombin group received a dose of purified antithrombin to reach an antithrombin activity value of $120 \%$, whereas patients in the control group did not receive antithrombin.
\end{abstract}

\begin{abstract}
Results: The antithrombin activity values were significantly higher in the antithrombin group at all postoperative determinations until discharge. Antithrombin activity levels $<58 \%$ at admission to the intensive care unit were found in $26.6 \%$ of patients in the control group versus none in the antithrombin group $(P=.001)$. Heparin resistance rate was significantly $(P=.001)$ higher in the control group $(38.2 \%)$ versus the antithrombin group $(17 \%)$. Patients in the antithrombin group had a significant but clinically irrelevant $(8 \mathrm{~mL} /$ hour $)$ higher postoperative bleeding, with no differences in transfusion rates. No differences were found for clinical outcomes, and no safety issues were identified.
\end{abstract}

Conclusions: Preoperative antithrombin supplementation prevents heparin resistance and avoids excessive postoperative decrease of antithrombin activity. (J Thorac Cardiovasc Surg 2013;145:1393-9)

Antithrombin (AT) supplementation in cardiac operations with cardiopulmonary bypass (CPB) is still an open issue. Low levels of AT before and during CPB are associated with a poor response to heparin, commonly defined as "heparin resistance" (HR), which occurs at a variable rate between $10 \%$ and $30 \%$ depending on the definition. ${ }^{1-4}$ The use of purified AT to treat HR has been suggested as an alternative to fresh frozen plasma administration. ${ }^{5}$ Four randomized controlled trials demonstrated the efficacy of purified AT for the treatment of $\mathrm{HR}$ before or during $\mathrm{CPB},{ }^{6-9}$ and the recently released update (2011) of the Society of Thoracic Surgeons and Society of Cardiovascular Anesthesiologists Guidelines on Blood Conservation Clinical Practice ${ }^{10}$ states that HR should be treated with AT concentrates before CPB (class IA).

During CPB, thrombin generation is mainly triggered by tissue factor released as a consequence of the surgical

\footnotetext{
From the Department of Cardiothoracic and Vascular Anesthesia and Intensive Care, ${ }^{\mathrm{a}}$ IRCCS Policlinico San Donato, Milan, Italy; Instituto Grifols S.A., ${ }^{\text {b }}$ Barcelona, Spain; and Scientific Directorate, ${ }^{\mathrm{c}}$ IRCCS Policlinico San Donato, Milan, Italy.

Supported by Instituto Grifols S.A. (Barcelona, Spain).

Disclosures: M.R. received honoraria from Instituto Grifols S.A., CSL Behring, Novo Nordisk, and Medtronic Inc for speaking at meetings and symposia. All other authors have nothing to disclose with regard to commercial support.

Received for publication June 26, 2012; revisions received Sept 6, 2012; accepted for publication Sept 21, 2012; available ahead of print Oct 29, 2012.

Address for reprints: Marco Ranucci, MD, FESC, Department of Anesthesia and Intensive Care, IRCCS Policlinico San Donato, Via Morandi 30, 20097 San Donato Milanese, Milan, Italy (E-mail: cardioanestesia@virgilio.it). $0022-5223 / \$ 36.00$

Copyright (c) 2013 by The American Association for Thoracic Surgery http://dx.doi.org/10.1016/j.jtcvs.2012.09.061
}

procedure and less importantly by contact with foreign surfaces. Heparin may only partially suppress thrombin generation, and the excessive hemostatic system activation may lead to coagulation factors consumption. Moreover, AT consumption during CPB may trigger postoperative thromboembolic complications, in presence of high thrombin generation. One observational study demonstrated an inverse relationship between postoperative AT levels and the incidence of a number of complications, including thromboembolic events, adverse neurologic events, bleeding, and prolonged intensive care unit (ICU) stay. ${ }^{11}$ Furthermore, guidelines ${ }^{10}$ place AT supplementation for the prevention of thromboembolic complications in selected patient populations in class IIB.

In the light of these arguments, it is reasonable to believe that preoperative supplementation with purified AT may represent a viable strategy for decreasing the hemostatic system dysregulation, preventing HR, and reducing postoperative morbidity in cardiac surgery operations with CPB.

This study is a phase II randomized controlled trial on pre-CPB purified AT supplementation aimed to investigate the effects of this treatment in maintaining postoperative AT values within the normal range, avoiding HR, and limiting postoperative complications.

\section{MATERIALS AND METHODS Study Design}

This study is a phase II single center, single blinded, randomized, controlled trial designed to evaluate preoperative AT supplementation in patients undergoing cardiac surgery with $\mathrm{CPB}$. The clinicians giving the 


\section{Abbreviations and Acronyms \\ $\mathrm{AE}=$ adverse event \\ $\mathrm{AT}=$ antithrombin \\ $\mathrm{CPB}=$ cardiopulmonary bypass \\ $\mathrm{HR}=$ heparin reistance \\ ICU $=$ intensive care unit \\ $\mathrm{SAE}=$ serious adverse event}

treatment were not blinded; patients and clinicians assessing outcome measurements were not aware of patient allocation.

The study was approved by the local ethics committee and national health authority and properly registered at a public trials registry, www. clinicaltrial.gov (trial ID NCT00823082). Written informed consent was obtained from all patients before their participation.

The study was supported by Instituto Grifols S.A. (Barcelona, Spain). Representatives were involved in designing the study but not in collecting, analyzing, or interpreting the data. They read and approved the final manuscript but had no influence in making the decision to submit for publication.

\section{Study Population}

Inclusion criteria were: male or female patient aged at least 18 years needing an elective heart surgery with CPB. To be eligible, subjects had to present a baseline AT activity $<100 \%$ and $>60 \%$ and be willing to comply with all aspects of the protocol, including blood sampling, for the total duration of the study.

Exclusion criteria included heart transplant operations, nonelective surgery, participation in another investigational study during the past 3 months, documented AT deficiency, history of anaphylactic reaction(s) to blood or blood components, allergies to excipients, and pregnancy.

From June 2009 to April 2011 patients undergoing elective cardiac surgery with CPB were considered for enrollment at a single institution (IRCCS Policlinico San Donato, Milan, Italy). One thousand ninety patients were evaluated for screening (Figure 1) of which 1407 patients did not meet the eligibility criteria (136 patients suffered from congenital heart disease, $60 \mathrm{pa}-$ tients had missing baseline AT level values, 16 had baseline AT levels $<60 \%$, 1056 had baseline AT activity $\geq 100 \%$, and 139 patients were enrolled in other randomized controlled trials), and 377 patients declined to participate or were not able to give consent because of their clinical condition. Screening, eligibility, and enrollment of patients are shown in Figure 1.

\section{Study Interventions}

The randomization scheme was electronically generated by an independent biostatistician and delivered to the center in sealed numbered envelopes.

The investigational drug (purified human plasma derived AT, Anbinex; Instituto Grifols S.A., Barcelona, Spain) was administered immediately after anesthesia induction as a single dose targeted to achieve a level of $120 \%$ according to the formula

$$
\mathrm{AT}(\mathrm{IU})=(120-\text { actual AT activity }) \times(\text { weight in } \mathrm{kg}) \times 0.8
$$

Patients randomized to the control group did not receive any dose of the study drug. Postoperative AT administration was excluded by the study protocol.

The value of $120 \%$ was decided based on the fact that the normal AT activity range is considered $80 \%$ to $120 \%,{ }^{12}$ and that using chromogenic tests the value of $120 \%$ is considered as the upper limit of the normal range by the majority of the manufacturers, including the one providing the assays used in this study.

\section{Intraoperative Anticoagulation}

Unfractionated heparin was intraoperatively administered before CPB to reach and maintain a target activated clotting time of 450 seconds during CPB.

The heparin loading dose was assessed using a heparin monitoring system (Medtronic Inc, Minneapolis, Minn), which provided the estimated dose to reach an activated clotting time of 450 seconds and the heparin sensitivity index (seconds/IU/mL) as a measurement of the patient's sensitivity to heparin. The latter tests were performed before administration of the study drug, and repeated in patients of the AT group after receiving the study drug. In the AT group, the heparin loading dose was established according to the post-AT administration test.

Further heparin doses during CPB were administered as a bolus of $100 \mathrm{IU} / \mathrm{kg}$ if needed to maintain the desired activated clotting time value.

Heparin was reversed with protamine sulphate at a 1:1 ratio with the heparin loading dose; a second protamine dose $(0.5 \mathrm{mg} / \mathrm{kg})$ was administered if required.

\section{AT Activity Determinations}

All AT activity determinations were performed in the same local hospital laboratory with a coagulometer Sysmex CA-6000 (Toa Medical Electronics, Milan, Italy) through a calibration curve prepared by serial dilution of a human normal plasma pool and expressed as percentage of activity.

\section{Data Collection and Time Points}

Clinical data collected during this study were the usual ones routinely collected in the institutional database: patient demographics, medical history, comorbidities, risk stratification according to the EuroSCORE, and intraoperative details (eg, type of operation, CPB and aortic crossclamp time duration, lowest hematocrit on $\mathrm{CPB}$, heparin and protamine dose, and heparin sensitivity index).

Laboratory data included complete blood count, biochemistry (eg, serum creatinine and liver enzymes) and coagulation tests (eg, AT activity, activated partial thromboplastin time, prothrombin time, international normalized ratio, and fibrinogen).

Throughout the study clinical data were collected at 8 separate time points: recruitment visit (within 14 days before the operation), preoperatively (the day of the operation), operatively (during the operation), at ICU admission, on postoperative days 1 and 2, at ICU discharge, and at the follow-up visit (1 month after the operation).

\section{Study Outcomes: Assessment of Efficacy}

The primary efficacy end points of the study were (1) AT activity levels at ICU admission higher in the AT group versus control group and (2) lower percentage of patients with AT activity $<58 \%$ in the AT group versus control group. The value of AT activity settled at $58 \%$ was not arbitrary, being based on our previous study ${ }^{11}$ where we could identify this cut-off value at the arrival in the ICU as the most specific and sensitive value predicting a prolonged ICU stay.

AT levels were measured at the recruitment visit and postoperatively immediately after the ICU admission, on postoperative days 1 and 2, and at discharge from the ICU.

Secondary efficacy end points were: HR (failure to reach an activated clotting time $>450$ seconds after a dose of up to $400 \mathrm{IU} / \mathrm{kg}$ heparin or to maintain this value despite heparin supplementation of $100 \mathrm{IU} / \mathrm{kg}$ ), blood loss (in the first postoperative 12 hours), number of plasma and packed red cells units needed during the ICU stay, mechanical ventilation duration (hours), ICU and hospital stay (days), and prolonged ( $>7$ days) ICU stay. Additional secondary end points were related to the postoperative complication rate (safety outcomes), which included surgical re-exploration (due to bleeding), low cardiac output syndrome (need for inotropic support $>48$ hours), myocardial infarction, adverse neurologic outcome, acute kidney injury (peak serum creatinine level $>2 \mathrm{mg} / \mathrm{dL}$ and twice the baseline), thromboembolic events (myocardial infarction, stroke, mesenteric infarction, or peripheral or pulmonary thromboembolism), and in-hospital mortality. 


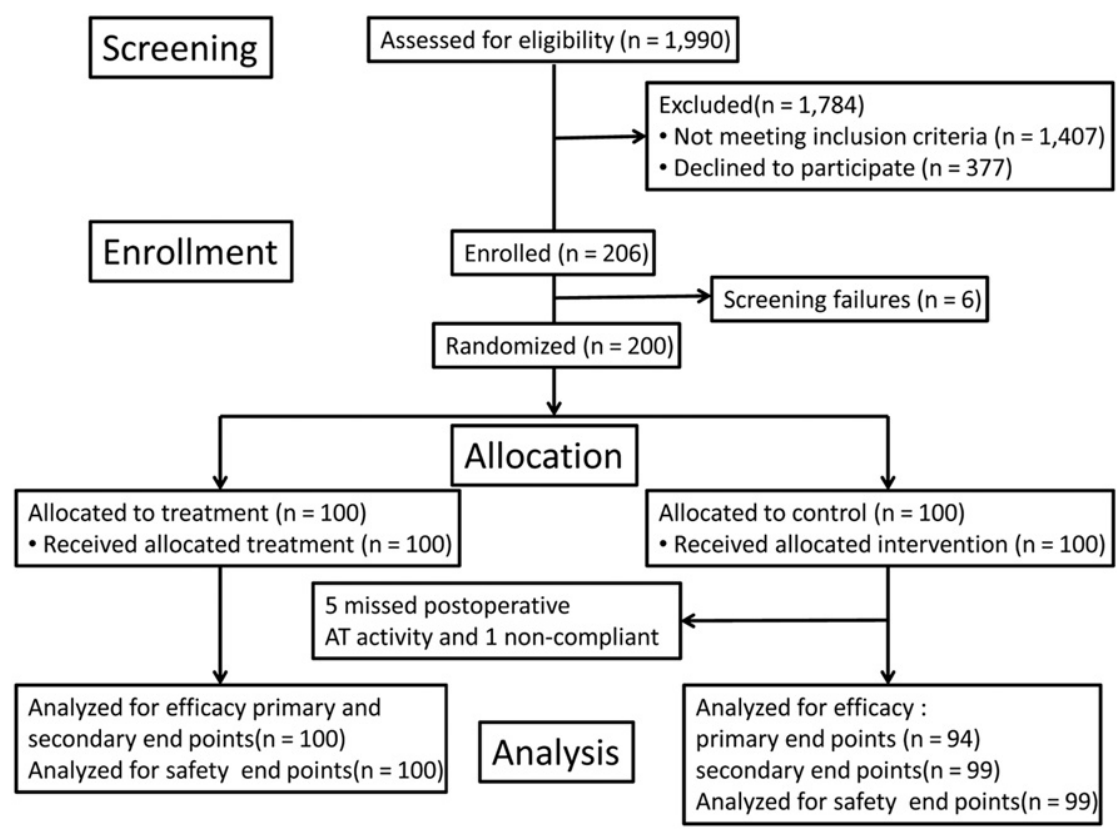

FIGURE 1. Flow diagram of enrollment, intervention allocation, and data analysis. AT, Antithrombin.

\section{Study Outcomes: Clinical Safety and Tolerability}

Clinical safety and tolerability of the investigational drug was assessed by monitoring vital signs, hematologic parameters, clinical chemistry parameters, and adverse events (AEs). Serious adverse events (SAEs) were defined as a medical occurrence that resulted in death, was lifethreatening, prolonged the hospitalization, or resulted in persistent or significant disability.

Viral safety included virology testing at the recruitment and follow-up visit.

\section{Statistical Analysis}

The sample size was calculated at 200 patients (100 patients per group), based on a $90 \%$ power to confirm the hypothesis that patients in the AT group would maintain a mean AT activity value $10 \%$ higher (in absolute value) than patients in the control group at the arrival in the ICU. This calculation was based on 2-sided tests, and predicted mean values and standard deviation of AT activity taken from previous studies. ${ }^{11,13}$

Continuous data are summarized by using descriptive statistics; that is, mean and standard deviation or median and range (minimum and maximum) according to their distribution. Categorical variables are summarized by using frequency (counts) and percentages, with relative risk and $95 \%$ confidence intervals when appropriate. Statistical tests applied in the efficacy and safety analysis included parametric (Student $t$ test, analysis of variance for repeated measures, and linear and binary regression analysis) and nonparametric tests (Mann-Whitney test). The primary end points were assessed with an analysis of variance for repeated measures (end point: AT activity higher in treatment arm) and relative risk analysis (lower rate of patients with $<58 \%$ AT activity at the arrival in the ICU in the treatment arm).

A receiver operating characteristics analysis with the area under the curve measurement was anticipated to assess the predictive value of postoperative AT activity in both treatment arms. Other statistical tests used for post hoc analyses included best fit between continuous variables using linear and nonlinear regression analyses.

\section{RESULTS}

The 2 groups' details are shown in Table 1. Patients in the AT group received a significantly lower dose of heparin to establish and maintain anticoagulation for $\mathrm{CPB}$, and a correspondent significantly lower dose of protamine to return to the baseline activated clotting time. Within the AT group the heparin sensitivity index significantly $(P<.001)$ increased after the administration of the study drug.

All patients who received the study drug were included in the analysis. For 5 patients in the control group the AT value at the arrival to the ICU was not determined. These patients were not included in the primary end points efficacy analysis but remained in the secondary end points efficacy analysis and safety analysis. One patient in the control group received a therapeutic dose of purified AT during the ICU stay according to medical judgment and was thus excluded from the efficacy analysis.

All patients completed the study protocol until ICU and hospital discharge. Follow-up at 1 month was completed by 146 patients $(75 \%$ of patients discharged from the hospital). This relatively low adherence to the 1-month follow-up was mainly due to patients living in regions other than Lombardy, who were unwilling to travel back to our institution.

Patients in the AT group received a median dose of 1800 IU (range, 1000-4000 IU) purified AT and the highest 90th percentile received was 3000 to $4000 \mathrm{IU}$.

\section{Efficacy Assessment}

Both of the primary efficacy end points were successfully reached. Postoperative AT values analyzed for repeated measures demonstrated a significant $(P=.002)$ between group difference in favour of the AT group. This effect was found at any postoperative point in time except for the follow-up visit (Figure 2). Twenty-five patients 
TABLE 1. Main pre- and intraoperative variables in the 2 groups

\begin{tabular}{|c|c|c|c|}
\hline $\begin{array}{c}\text { Variable } \\
\text { No. or mean } \pm \text { SD }\end{array}$ & $\begin{array}{l}\text { Control group } \\
\quad(\mathbf{n}=\mathbf{1 0 0})\end{array}$ & $\begin{array}{l}\text { AT group } \\
(\mathbf{n}=\mathbf{1 0 0})\end{array}$ & $\begin{array}{c}P \\
\text { value }\end{array}$ \\
\hline Age $(y)$ & $67.8 \pm 11.2$ & $66.9 \pm 10.5$ & .890 \\
\hline Male gender & 75 & 83 & .224 \\
\hline Weight $(\mathrm{kg})$ & $78.3 \pm 17.3$ & $80.5 \pm 17.5$ & .374 \\
\hline Hypertension & 86 & 71 & .009 \\
\hline Hypercholesterolemia & 56 & 47 & .203 \\
\hline $\begin{array}{l}\text { Left ventricular ejection } \\
\text { fraction }(\%)\end{array}$ & $53.9 \pm 9.6$ & $54.3 \pm 10.7$ & .766 \\
\hline $\begin{array}{l}\text { Recent }(30 \mathrm{~d}) \text { myocardial } \\
\text { infarction }\end{array}$ & 20 & 10 & .073 \\
\hline Unstable angina (current) & 5 & 11 & .613 \\
\hline $\begin{array}{l}\text { Congestive heart failure } \\
\text { (current) }\end{array}$ & 2 & 3 & .651 \\
\hline Active endocarditis & 1 & 1 & 1.000 \\
\hline Chronic dialysis & 1 & 0 & .316 \\
\hline $\begin{array}{l}\text { Chronic obstructive } \\
\text { pulmonary disease }\end{array}$ & 8 & 13 & .357 \\
\hline Previous neurologic accident & 5 & 4 & .733 \\
\hline $\begin{array}{l}\text { Previous pulmonary } \\
\text { embolism }\end{array}$ & 2 & 2 & 1.000 \\
\hline Peripheral vascular disease & 13 & 12 & .831 \\
\hline $\begin{array}{l}\text { Previous cardiovascular } \\
\text { surgery }\end{array}$ & 5 & 6 & .756 \\
\hline Diabetes on medication & 21 & 28 & .324 \\
\hline Severe liver disease & 2 & 3 & .651 \\
\hline Logistic EuroSCORE & $5.9 \pm 4.7$ & $5.3 \pm 5.2$ & .459 \\
\hline Oral anticoagulant therapy & 17 & 16 & .849 \\
\hline Heparin therapy (current) & 25 & 29 & .633 \\
\hline Serum creatinine $(\mathrm{mg} / \mathrm{dL})$ & $1.04 \pm 0.82$ & $1.02 \pm 0.35$ & .814 \\
\hline Hemoglobin (mg/dL) & $12.6 \pm 1.65$ & $12.5 \pm 1.56$ & .545 \\
\hline $\begin{array}{l}\text { Platelet count } \\
\qquad(\times 1000 \text { cells } / \mu \mathrm{L})\end{array}$ & $191 \pm 55$ & $185 \pm 62$ & .475 \\
\hline Fibrinogen $(\mathrm{mg} / \mathrm{dL})$ & $337 \pm 103$ & $311 \pm 88$ & .062 \\
\hline $\begin{array}{l}\text { Activated partial } \\
\text { thromboplastin time (s) }\end{array}$ & $31.1 \pm 4.4$ & $32.8 \pm 9.5$ & .108 \\
\hline International normalized ratio & $1.17 \pm 0.14$ & $1.18 \pm 0.13$ & .602 \\
\hline Isolated coronary operation & 50 & 40 & .155 \\
\hline Isolated valve operation & 31 & 26 & .436 \\
\hline Coronary + valve operation & 12 & 20 & .127 \\
\hline Others & 7 & 14 & .112 \\
\hline $\mathrm{CPB}$ duration (min) & $83.2 \pm 33.6$ & $84.1 \pm 35.6$ & .858 \\
\hline $\begin{array}{l}\text { Aortic crossclamp } \\
\text { duration (min) }\end{array}$ & $58.4 \pm 29.5$ & $60.8 \pm 30.2$ & .583 \\
\hline $\begin{array}{l}\text { Total heparin dose } \\
\qquad \times 100 \mathrm{IU})\end{array}$ & $271 \pm 85$ & $220 \pm 67$ & .001 \\
\hline Total protamine dose $(\mathrm{mg})$ & $268 \pm 69$ & $227 \pm 57$ & .001 \\
\hline $\begin{array}{l}\text { Lowest hematocrit on } \\
\text { CPB }(\%)\end{array}$ & $26.3 \pm 4.1$ & $27.0 \pm 3.2$ & .158 \\
\hline HSI (baseline) (sec/IU/mL) & $94.8 \pm 24$ & $95.5 \pm 29$ & .895 \\
\hline $\begin{array}{l}\text { HSI after purified AT } \\
(\mathrm{s} / \mathrm{IU} / \mathrm{mL})\end{array}$ & Not measured & $120 \pm 41$ & $.001 *$ \\
\hline
\end{tabular}

SD, Standard deviation; $A T$, antithrombin; EuroSCORE, European System for Cardiac Operative Risk Evaluation; $C P B$, cardiopulmonary bypass; HIS, heparin sensitivity index. *Within the AT group, after vs before purified AT administration.

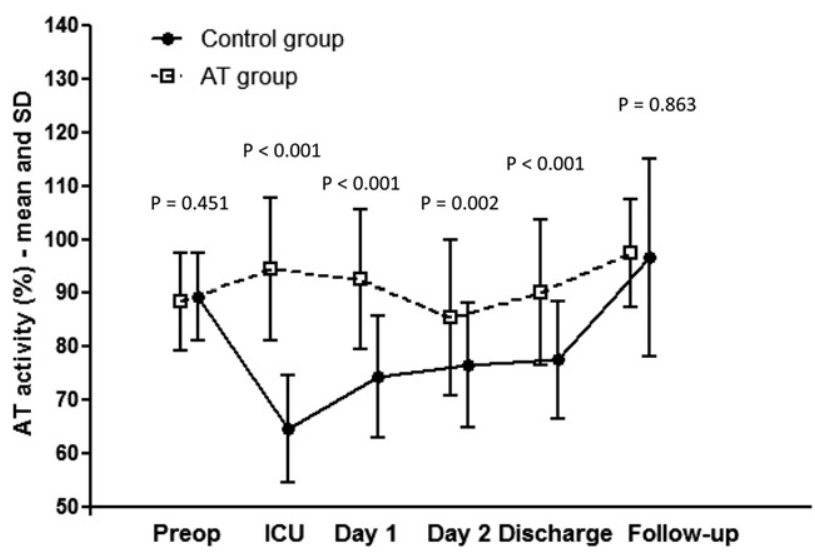

FIGURE 2. Antithrombin $(A T)$ activity levels during the study period. $S D$, Standard deviation; $I C U$, intensive care unit.

$(26.6 \%)$ in the control group had an AT activity level $<58 \%$ versus none $(0 \%)$ in the AT group $(P<.001)$.

The secondary efficacy and safety end points evaluated during the entire follow-up period are depicted in Figure 3. Patients in the AT group had a significantly $(P<.001)$ lower rate of heparin resistance. No significant differences were found with respect to the total or specific complications rates for binary events. There were 3 adverse neurologic events in the AT group ( 1 anoxic coma postcardiac arrest in the ward and 2 cerebral hemorrhages) and no events in the control group. These differences were not statistically significant.

Patients in the AT group (Table 2) had a significantly higher postoperative bleeding that was of little clinical relevance ( $8 \mathrm{~mL} /$ hour); no differences were found with respect to the number of packed red cells, fresh frozen plasma, and platelet concentrates units transfused. Duration of mechanical ventilation, ICU stay, and overall hospital stay were not significantly different between study groups.

Overall, there was a trend toward an increased incidence of complications in the AT group (22 out of 99 vs 31 out of 100).

\section{Safety Assessment}

There were 528 AEs in the AT group and 443 in the control group. A total of 64 SAEs in 32 patients occurred in the AT group and 51 in 29 patients in the control group. Three AEs/SAEs were considered as potentially related to the study drug, of which 2 were rated as SAEs: 1 case of cerebral hemorrhage (cause-effect relationship: doubtful); and 1 case of hepatitis $\mathrm{C}$ virus seroconversion (cause-effect relationship: possible). In the latter case an indeterminate ('doubtful') positive result for hepatitis $C$ virus antibody was found at the follow-up visit after a negative baseline hepatitis $\mathrm{C}$ virus antibody result. Although the patient did not receive any transfusions during surgery he did present other important risk factors that could explain this seropositivity: surgery itself and a close relative (wife) that was hepatitis $\mathrm{C}$ virus positive. 


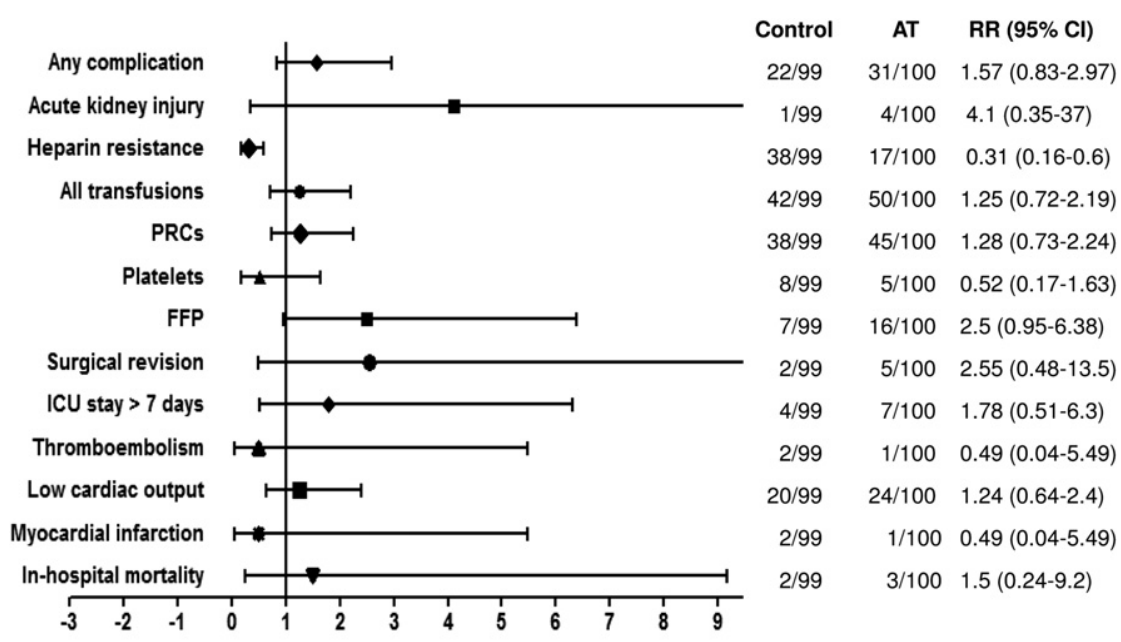

Favors AT

Favors control

FIGURE 3. Secondary end points (clinical outcomes). Relative risk $(R R)$ with $95 \%$ confidence intervals $(95 \%$ CIs). $A T$, Antithrombin; $P R C$, packed red cells; FFP, fresh frozen plasma; ICU, intensive care unit.

\section{Post Hoc Analysis: AT Activity at the Arrival to the ICU and Postoperative Outcome}

We conducted a post hoc analysis to determine the relationship between AT activity immediately at the arrival to the ICU and the length of ICU stay in our study population, taking into account the treatment arm.

In Figure 4 the association between ICU length of stay and AT activity at the arrival to the ICU is depicted. Different nonlinear regression analyses were performed to identify the best fit for the relationship. Given the non-normal distribution of the ICU stay variable, a Poisson weighting was applied to the different models tested. The nonlinear equation with the best fit was a quadratic, U-shaped equation, where the length of ICU stay is dependent on the AT activity according to the equation:

$$
\begin{aligned}
\operatorname{ICU} \operatorname{stay}(\text { days })= & 12.72-0.28 \times \text { AT activity } \\
& +0.0018 \times(\text { AT activity })^{2}
\end{aligned}
$$

where the weighted $R^{2}$ was 0.165 ( $P$ value for association $<.001)$.

TABLE 2. Outcome parameters (continuous variables)

\begin{tabular}{lccc}
\hline $\begin{array}{c}\text { Variable (median and } \\
\text { range) }\end{array}$ & $\begin{array}{c}\text { Control group } \\
(\mathbf{n = 9 9 )}\end{array}$ & $\begin{array}{c}\text { AT group } \\
(\mathbf{n}=\mathbf{1 0 0})\end{array}$ & $\begin{array}{c}\boldsymbol{P} \\
\text { value }\end{array}$ \\
\hline Blood loss (mL/12 h) & $350(100-1875)$ & $450(50-1950)$ & .011 \\
No. of PRCs units transfused & $0(0-11)$ & $0(0-10)$ & .304 \\
No. of FFP units transfused & $0(0-9)$ & $0(0-6)$ & .106 \\
No. of PLT units transfused & $0(0-13)$ & $0(0-5)$ & .379 \\
Mechanical ventilation (h) & $12(4-110)$ & $15(3-450)$ & .367 \\
ICU stay (d) & $2(1-13)$ & $2(1-22)$ & .372 \\
Hospital stay (d) & $7(4-27)$ & $7(4-32)$ & .556 \\
\hline
\end{tabular}

AT, Antithrombin; $P R C s$, packed red cells; $F F P$, fresh frozen plasma; $P L T$, platelet concentrates; $I C U$, intensive care unit.
Patients in the control group had a significant negative association of the AT activity at the arrival in the ICU with the presence of a prolonged ( $>7$ days) ICU stay. An receiver operating characteristics analysis (Figure 5) demonstrated the predictive value of the AT activity at the arrival in the ICU for prolonged ICU stay, with an area under the

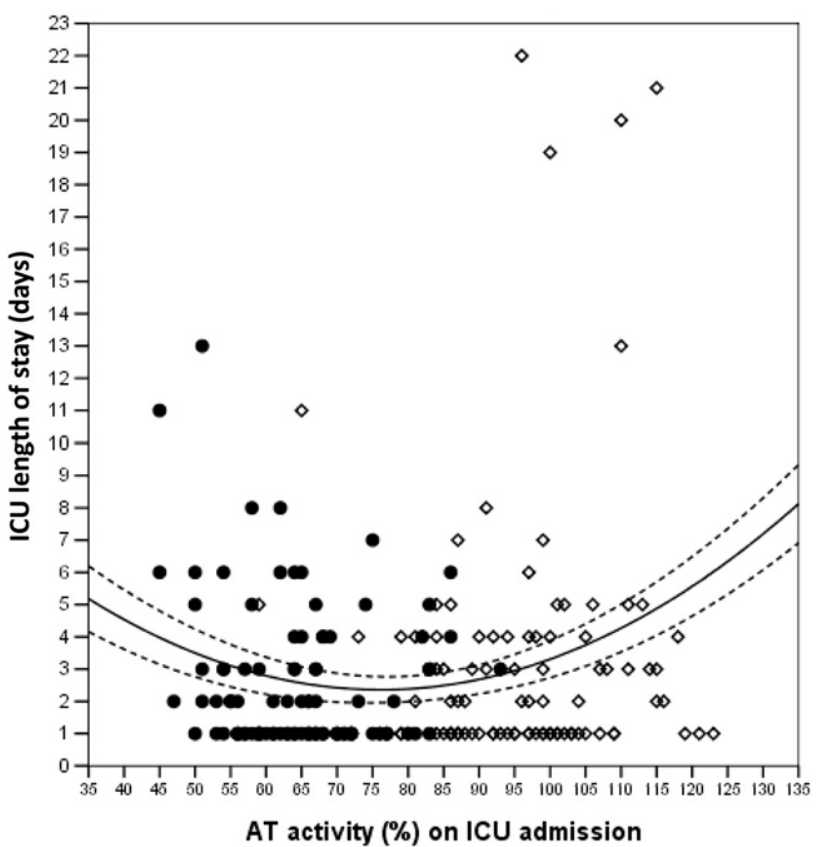

FIGURE 4. Scatterplot showing the relationship between intensive care unit $(I C U)$ length of stay and antithrombin (AT) activity at ICU admission. Solid line represents the quadratic regression fit of the relationship and the broken line its $95 \%$ confidence limits. Open diamonds, AT group; black dots, control group. 
curve of 0.821 and a cut-off value settled at an AT activity $<58 \%$ (75\% sensitivity and $73 \%$ specificity).

\section{DISCUSSION}

This study fulfilled the primary efficacy end points demonstrating that (1) preoperative supplementation with purified AT maintains AT activity at a significantly higher level than untreated patients upon ICU admission and during the first 2 postoperative days, and (2) no patients treated with purified AT experienced low $(<58 \%)$ levels of AT activity during the postoperative course. Additional findings (secondary end points) are that the heparin resistance rate is significantly lower in AT-treated patients, that lower doses of heparin were required to reach and maintain an activated clotting time $>450$ seconds in AT-treated patients, and that AT supplementation significantly increased the heparin sensitivity of these patients.

No significant differences with respect to postoperative outcomes were observed between the 2 study groups, except a significant, but clinically irrelevant higher postoperative bleeding in AT-treated patients. This study did not raise safety issues and the rate of AEs/SAEs was consistent with the expected findings in this patient population. However, there was a trend toward a higher rate of complications in the AT-treated patients that may deserve further attention.

AT supplementation in heparin-resistant patients is already established as a first-choice treatment ${ }^{6-10}$ preferred over fresh frozen plasma. The novelty of our study is the pre-emptive use of purified AT, aimed to maintain the postoperative AT activity at a normal value.

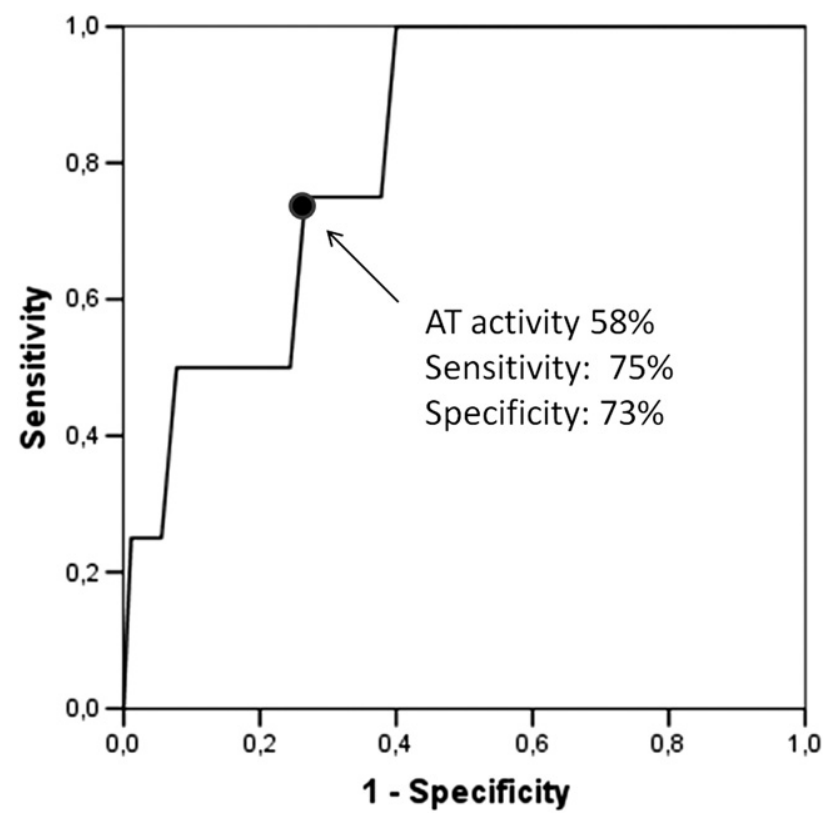

FIGURE 5. Receiver operating characteristic analysis for antithrombin $(A T)$ activity at the intensive care unit admission as a predictor of prolonged ( $>7$ days) intensive care unit stay in control group patients.
Previous observational studies have highlighted that low levels of AT activity at the arrival to the ICU and during the first postoperative days were associated with bad outcomes ${ }^{11}$ and major adverse cardiovascular events. ${ }^{14}$ Our phase II randomized controlled study was not powered to detect clinical differences in postoperative outcomes and major adverse cardiovascular events between the 2 arms. However, a trend toward fewer thromboembolic events was found in the AT group, at the expense of a slightly higher bleeding tendency.

Previous studies have already raised the concern that AT supplementation may result in a higher postoperative bleeding tendency. ${ }^{13}$ This is not surprising given the well-known anticoagulant properties of AT.

Some additional information may come from the within group analysis conducted in this trial, focused on the association between AT activity at ICU admission and length of ICU stay. Lacking the power for specific clinical outcomes assessment, the length of ICU stay may be considered as a surrogate for a composite number of moderate to severe complications that result in a prolonged ICU stay, as already demonstrated in a previous study. ${ }^{11}$

The analysis of patients in the control group confirms previous findings that the lower the AT activity level at ICU admission is, the higher the risk for a prolonged ( $>7$ days) ICU stay is. This partially contradicts the findings of other studies, ${ }^{14}$ again strengthening the hypothesis that low AT values anticipate, and do not follow the AEs. Interestingly, the critical value of AT activity $<58 \%$ at ICU admission was confirmed in this study.

Some of the patients in the AT group demonstrated a prolonged ICU stay with high values ( $>95 \%$ ) of AT activity at ICU admission. This trend introduces the hypothesis that some of the AT-treated patients may have experienced an AT overcorrection. Despite the application of an equation based on weight and preoperative AT activity, there were some patients with probably excessive postoperative AT activity values, owing to the administration of a higher AT dose. However, our AT-treated patients did not receive unusually high AT doses, with only $10 \%$ receiving $>3000$ units, a dose lower than those used in previous studies using purified $(50 \mathrm{IU} / \mathrm{kg})^{15}$ or recombinant AT $(75 \mathrm{IU} / \mathrm{kg}){ }^{7,8}$ Therefore, this study raises the hypothesis that a preoperative target value of $120 \%$ may be excessive, especially in absence of the recognized factors increasing AT consumption (eg, CPB duration, opening of the cardiac chambers, and advanced age). The achievement of this potentially excessive target value may be responsible for the trend towards a higher complication rate (namely bleeding related) in the AT group.

The overall analysis of the U-shaped relationship between AT activity at ICU admission and ICU length of stay highlights that patients with shorter ICU stays belong to an area with AT activity between $70 \%$ and $95 \%$ at ICU admission, either spontaneously reached by untreated 
patients or achieved through AT supplementation in treated patients. This range may probably represent a correct balance between the risk of bleeding and the risk of thrombotic events. Values below this range may probably place the patient in an area at risk for excessive thrombin generation and micro/macrovascular thrombosis; conversely, values above this range are associated with a more pronounced risk of bleeding events.

\section{Study Limitations}

There are some limitations in this randomized, controlled trial. First of all, due to the need for adjusting the heparin dose to the AT activity, the study was not double-blinded. Moreover, given the fact that postoperative AT activity is routinely measured in our institution, it was not possible to blind the clinicians in charge for the postoperative course. Finally, the follow-up was not completed by a considerable amount of patients. This was mainly due to the presence of many patients referred from external, out-of-region hospitals.

\section{Future Perspectives}

This phase II trial may open the possibility to run a phase III trial powered to address more clinically relevant end points (ie, major morbidity and mortality). Moreover, we think that our study may offer a useful advice for the design of future trials, which should avoid "overcorrection" of AT values, which probably induced some of the observed complications in the AT-treated patients. A more reasonable target for preoperative AT activity could be not higher than $100 \%$.

Alternatively, future trials may focus more on patients with a lower preoperative AT activity (ie, $<80 \%$ ), or at higher risk to experience a huge intraoperative loss of AT activity (ie, prolonged CPB $>120$ minutes).

In conclusion, our study confirms the role of preoperative AT supplementation for the prevention of heparin resistance, demonstrating that this strategy avoids that the patients may experience very low postoperative AT activity values (that are associated with a prolonged ICU stay). We believe that our data provide clinically relevant information for further phase III studies focused on clinical outcomes, particularly as a guide for the choice of the adequate purified AT dose.

\section{References}

1. Ranucci M, Isgrò G, Cazzaniga A, Soro G, Menicanti L, Frigiola A. Predictors for heparin resistance in patients undergoing coronary artery bypass grafting. Perfusion. 1999; 14:437-42.

2. Ranucci M, Isgrò G, Cazzaniga A, Ditta A, Boncilli A, Cotza M, et al. Different patterns of heparin resistance: therapeutic implications. Perfusion. 2002;17 199-204.

3. Rodríguez-López JM, del Barrio E, Lozano FS, Muriel C. Does preoperative level of antithrombin III predict heparin resistance during extracorporeal circulation? Anesth Analg. 2008;107:1444-5.

4. Muedra V, Bonanad S, Gomez M, Villalonga V, Sanchez F, LLopis JE. Relationships between antithrombin activity, anticoagulant efficacy of heparin therapy and perioperative variables in patients undergoing cardiac surgery requiring cardiopulmonary bypass. Perfusion. 2011;26:487-95.

5. Spiess B. Treating heparin resistance with antithrombin or fresh frozen plasma. Ann Thorac Surg. 2008;85:2153-60.

6. Williams MR, D'Ambra AB, Beck A, Spanier TB, Morales DL, Helman DN et al. Randomized trial of antithrombin concentrate for treatment of heparin resistance. Ann Thorac Surg. 2000;70:873-7.

7. Avidan MS, Levy JH, Scholz J, Delphin E, Rosseel PM, Howie MB, et al. A phase III, double-blind, placebo-controlled, multicenter study on the efficacy of recombinant human antithrombin in heparin-resistant patients scheduled to undergo cardiac surgery necessitating cardiopulmonary bypass. Anesthesiology. 2005; 102:276-84.

8. Avidan MS, Levy JH, van Aken H, Feneck RO, Latimer RD, Ott E, et al. Recombinant human antithrombin III restores heparin responsiveness and decreases activation of coagulation in heparin-resistant patients during cardiopulmonary bypass. J Thorac Cardiovasc Surg. 2005;130:107-13.

9. Lemmer JH Jr, Despotis GJ. Antithrombin III concentrate to treat heparin resistance in patients undergoing cardiac surgery. J Thorac Cardiovasc Surg. 2002 123:213-7.

10. Society of Thoracic Surgeons Blood Conservation Guideline Task Force, Ferraris VA, Brown JR, Despotis GJ, Hammon JW, Reece TB, Saha SP, et al. 2011 Update to The Society of Thoracic Surgeons and the Society of Cardiovascular Anesthesiologists blood conservation clinical practice guidelines. Ann Thorac Surg. 2011;91:944-82.

11. Ranucci M, Frigiola A, Menicanti L, Ditta A, Boncilli A, Brozzi S. Postoperative antithrombin levels and outcome in cardiac operations. Crit Care Med. 2005;33: 355-60.

12. Thaler E, Lechner K. Antithrombin deficiency and thromboembolism. Clin Haematol. 1981;10:369-90.

13. Rossi M, Ranucci M, Soro G, Schiavello R, Guarneri S. Purified antithrombin supplementation in coronary revascularization operations. Eur J Anaesth 2007;24(suppl 40):71-6.

14. Garvin S, Muehlschlegel JD, Perry TE, Chen J, Liu KY, Fox AA, et al. Postoperative activity, but not preoperative activity, of antithrombin is associated with major adverse cardiac events after coronary artery bypass graft surgery. Anesth Analg. 2010;111:862-9.

15. Koster A, Fischer T, Gruendel M. Management of patients with heparin resistance during cardiopulmonary bypass: the effect of five different anticoagulation strategies on hemostatic activation. J Cardiothorac Vasc Anesth. 2003;17:171-5. 